\title{
A Novel Approach to Inactivate the Clinical Isolates of Trichophyton mentagrophytes and Trichophyton rubrum by Using Non-Thermal Plasma
}

Anser Ali ${ }^{1,2 \dagger}$, Young June Hong ${ }^{2,3 \dagger}$, Jihoon Park ${ }^{2,3}$, SeungHyun Lee ${ }^{1,2}$, Eun Ha Choi ${ }^{1-3}$, Gi Chung Kwon ${ }^{2,3 *}$ and Bong Joo Park ${ }^{2,3 *}$

${ }^{1}$ Department of Plasma Bioscience and Display, Kwangwoon University, Republic of Korea

${ }^{2}$ Plasma Bioscience Research Center, Kwangwoon University, Republic of Korea

${ }^{3}$ Department of Electrical and Biological Physics, Kwangwoon University, Republic of Korea

tThese authors contributed equally to this work

\begin{abstract}
In this study, we propose a novel approach that uses non-thermal plasma to inactivate dermatophytes, and we evaluate the antifungal activity of the proposed method using an In vitro infected skin model with clinical isolates of dermatophytes. For this study, a floating electrode-dielectric barrier discharge (FE-DBD) plasma jet was the source for non-thermal plasma, and we investigated its antifungal activity on Trichophyton mentagrophytes ( $T$. mentagrophytes) and Trichophyton rubrum (T. rubrum), two clinical isolates of dermatophytes. These two strains are the most frequently isolated from skin, hair, and nail infections for use in an infected skin model.

The results of this study confirm that the FE-DBD plasma jet has statistically significant antifungal activity in saline and in an infected skin model, and after plasma treatment; we also observed a significant inhibition of the growth of the conidia and hypha for both strains in the infected skin mimicking model. In addition, we found a time-dependent correlation between the intracellular reactive species and the fungal cell viabilities after exposure to plasma. These results indicate FE-DBD plasma can inactivate clinically isolated dermatophyte species and suggest that non-thermal plasma may be an effective tool for use in supportive and/or alternative treatments for dermatophytosis.
\end{abstract}

Keywords: Floating Electrode-Dielectric Barrier Discharge (FE-DBD) Plasma; Dermatophytes; Trichophyton mentagrophytes; Trichophyton rubrum; Inactivation; In vitro infected skin model

\section{Introduction}

Dermatophytosis is one of the most common and widespread infectious skin diseases, and it is a significant public health problem. Skin mycosis now affects more than $20-25 \%$ of the world's population, and this high prevalence makes the condition one of the most frequent forms of skin infection. This kind of disease can be caused by dermatophytes, a type of fungi that produce an infection by invading keratinized animal tissue (including that of humans) such as skin, nail, and hair. The most common infections caused by dermatophytes are those that involve members of the Trichophyton genus, such as Trichophyton mentagrophytes (T. mentagrophytes) and Trichophyton rubrum (T. rubrum) [1-3].

Most cases are treated with topical antifungal agents, such as itraconazole, griseofulvin, or terbinafine, except for cases involving severe infections that cover an extensive area or with strains that are resistant to treatment. Drugs are often used systematically $[4,5]$, but prolonged treatment to these drugs may affect the liver, so it is necessary to periodically monitor liver function [6]. A further complication is that these drugs may interact and interfere with other medications [4]. Therefore, a new, alternative antifungal therapy is required that has high efficacy, reduced risk of side-effects, and lower cost. Consequently, there is a growing interest to develop alternative therapies, such as that involving non-thermal plasma, to combat these practical problems and to improve the quality of treatment.

In recent years, several reports have discussed in vitro antimicrobial activity of non-thermal plasma. Non-thermal plasma has been considered for use in clinical applications, including the treatment of diverse skin and soft tissue infections, since it produces ions, radicals, and various reactive species that inactivate microorganisms. Furthermore, non-thermal plasmas can operate at a low cost with high efficacy and are easy to handle. Most importantly, they do not leave toxic conditions after treatment [7-12].

Therefore, we investigate here the antifungal activity of a nonthermal floating electrode-dielectric barrier discharge (FE-DBD) plasma jet against clinical isolates of $T$. mentagrophytes and $T$. rubrum. These two strains are major pathogens responsible for nail and skin infections, and we test this novel approach for dermatophyte inactivation using an in vitro infected skin mimicking model.

\section{Materials and Methods}

\section{Microorganism}

Two clinical isolates of dermatophytes, T. mentagrophytes and T. rubrum, were obtained from the Hygiene and Microbiology Research Center in Japan. The strains were grown on potato dextrose agar (PDA, Becton, Dickinson and Company, Sparks, MD, USA) and sabouraud dextrose agar (SDA; Becton, Dickinson and Company, Sparks, MD, USA) at $25^{\circ} \mathrm{C}$ in aerobic conditions for 10 to 14 days before use.

*Corresponding authors: Gi Chung Kwon, Department of Electrical and Biologica Physics and Plasma Bioscience Research Center, Kwangwoon University, 20 Kwangwoon-gil, Nowon-gu, Seoul 139-701, Republic of Korea, Tel: +82-2-9405479; Fax: +82-2-940-5664; E-mail: gckwon@kw.ac.kr

Bong Joo Park, Department of Electrical and Biological Physics and Plasma Bioscience Research Center, Kwangwoon University, 20 Kwangwoon-gil, Nowongu, Seoul 139-701, Republic of Korea, Tel: +82-2-940-8629; Fax: +82-2-940-5664; E-mail: parkbj@kw.ac.kr

Received June 17, 2014; Accepted July 05, 2014; Published July 11, 2014

Citation: Ali A, Hong YJ, Park J, Lee SH, Choi EH, et al. (2014) A Novel Approach to Inactivate the Clinical Isolates of Trichophyton mentagrophytes and Trichophyton rubrum by Using Non-Thermal Plasma. J Microb Biochem Technol 6: 314-319. doi:10.4172/1948-5948.1000161

Copyright: (C) 2014 Ali A, et al. This is an open-access article distributed under the terms of the Creative Commons Attribution License, which permits unrestricted use, distribution, and reproduction in any medium, provided the original author and source are credited 
Citation: Ali A, Hong YJ, Park J, Lee SH, Choi EH, et al. (2014) A Novel Approach to Inactivate the Clinical Isolates of Trichophyton mentagrophytes and Trichophyton rubrum by Using Non-Thermal Plasma. J Microb Biochem Technol 6: 314-319. doi:10.4172/1948-5948.1000161

\section{Non-thermal FE-DBD plasma jet plasma device}

The FE-DBD plasma jet used in this study consists of a flexible plasma outlet tube, an inlet for gas injection and a needle electrode covered by a cylindrical glass tube (Figure 1a). Argon is used as a working gas and was injected from the gas inlet into a flexible plasma outlet tube at the rate of 2 liters per minute $(\mathrm{l} \mathrm{pm})$. As shown in Figure 1a and $1 \mathrm{~b}$, the substrate contacted by the plasma acts as an electrical ground. To generate atmospheric plasma, the FE-DBD jet was supplied with a root mean squared voltage of $8 \mathrm{kV}$ and current of around 33 $\mathrm{mA}$. To investigate the basic plasma parameters, the optical emission spectrum (OES) was measured by using a charge-coupled device (CCD) spectrometer (Ocean optics, model HR-4000) that can obtain emission spectra from 200 to $1100 \mathrm{~nm}$ wavelengths. The electron temperature of our plasma jet was estimated to be of about $0.7 \mathrm{eV}$ by investigating the argon emission lines (2p-1s). In addition, the electrical power input was calculated to be of $49 \mathrm{~W}$, and the sample temperature at a maximum treatment time (30 minutes) with this device was observed to be below $40^{\circ} \mathrm{C}$

\section{Conidia inactivation of dermatophytes in saline}

To examine the inactivation of T. mentagrophytes and T. rubrum by the plasma, we used a method described by Liu et al. [13]. Briefly, their conidia were cultured on PDA and SDA at $25^{\circ} \mathrm{C}$ in aerobic conditions for 10 days. The conidia from fresh culture were re-suspended in saline to obtain 1 to $5 \times 10^{6}$ cells $/ \mathrm{ml}$ as a working solution. Then, $1 \mathrm{ml}$ of working solution was treated for $0,5,10,20$, and 30 minutes by the FE-DBD plasma jet in a 24 well plate (Crystal grade polystyrene, gamma sterilized, SPL Korea), keeping an untreated sample as control. Finally, $100 \mu \mathrm{l}$ samples for each treatment time were spread on PDA and SDA and were incubated for 7 days. Colony forming units (CFU) were counted to evaluate the effect of the plasma on the conidia of both strains.

\section{Conidia and hypha inactivation of dermatophytes in infected skin mimicking condition}

To demonstrate a clear visual outcome of the plasma treatment,

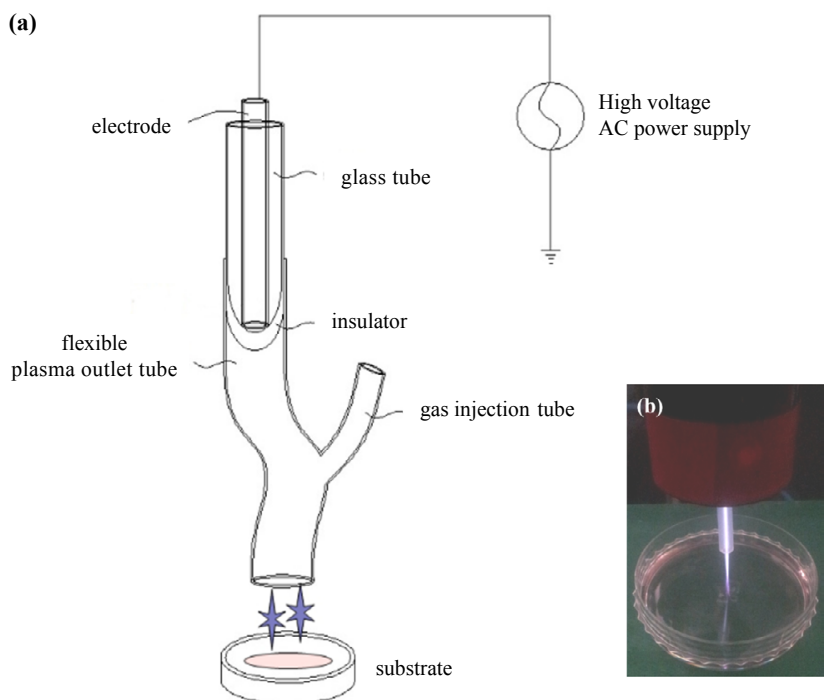

Figure 1: FE-DBD plasma jet device. (a) Schematic diagram of an FE-DBD plasma jet. (b) FE-DBD plasma jet treatment on a sample. Argon was used as the input gas throughout the experiments with a $2 \mathrm{lpm}$ flow rate.
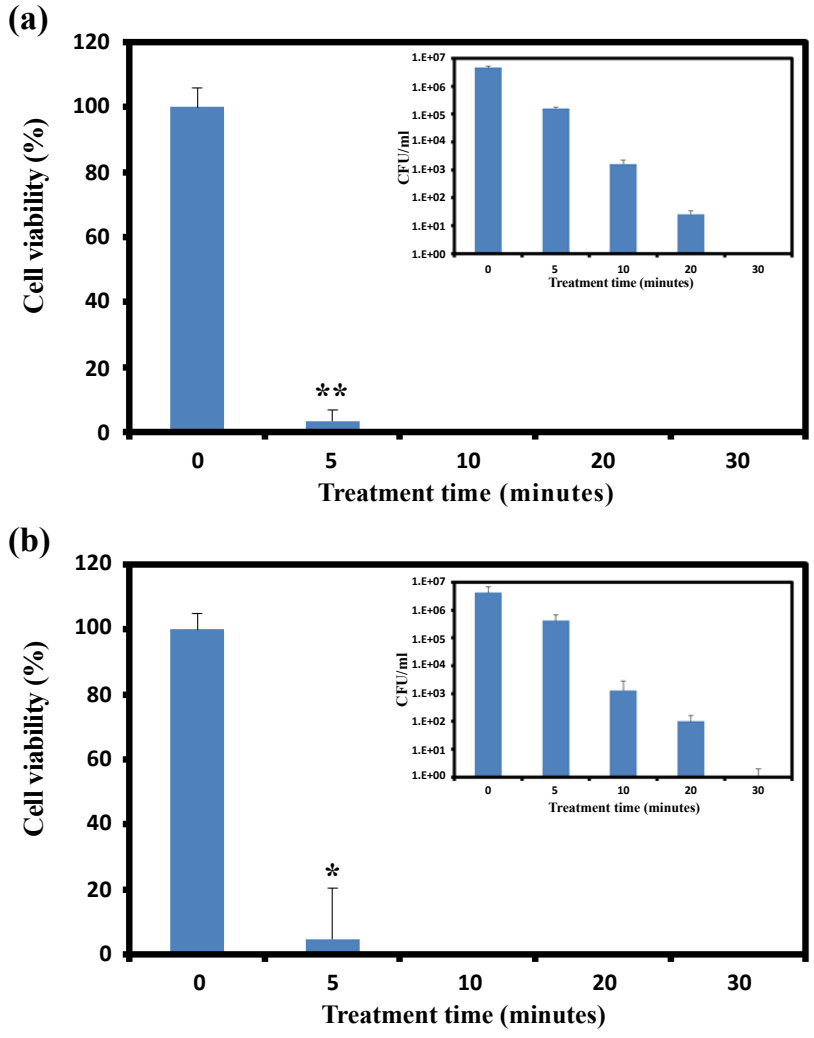

Figure 2: Fungal cell viabilities in saline after exposure to plasma. (a) Cell viability of $T$. mentagorophyte and (b) T. rubrum. The inserts in each figure represents the CFU values of each strain after treatment with plasma. The fungal conidia were treated with plasma for $0,5,10,20$, and 30 minutes. All data are expressed as a mean \pm standard deviation and were analyzed via Student's t-tests. Statistical significance was considered as $p<0.05\left(^{*}, p<0.05\right.$; ${ }^{* *}, p<0.005$ vs. control at the same time).

a qualitative test was performed with conidia and hypha state of $T$. mentagrophytes and T. rubrum according to the method of Fridman et al. [9] with some modifications. We attempted to mimic the structure of the skin and developed a skin model infected with fungi using agar (Figures $3 \mathrm{a}$ and $3 \mathrm{~b}$ ). For this analysis, a working solution of fungal conidia was made with saline at a concentration of 1 to $5 \times 10^{6}$ cells/ $\mathrm{ml}$. Then, $400 \mu \mathrm{l}$ of working solution were mixed with $3.6 \mathrm{ml}$ liquid PDA or SDA, and the mixture was thoroughly spread on each PDA and SDA culture plate to make an infected epidermis condition with fungal conidia. The conidia samples were treated with plasma for 0,3 , 5 , and 10 minutes, keeping an untreated sample as control. For making an infected epidermis condition with fungal hypha, the PDA and SDA conidia plates were incubated for 3 days at $25^{\circ} \mathrm{C}$ to allow the conidia to develop into hypha before treatment. After incubation for 3 days, the developed hypha inside the PDA and SDA were also treated with plasma for $0,3,5$, and 10 minutes. All samples were photographed and the diameter of growth inhibition areas was measured in each plate on the seventh day of incubation to assess the effect of the plasma treatment.

\section{Detection of live and dead cells after plasma treatment}

A fluorescence-based live-dead cell assay was performed to evaluate the viability of the fungal cells. Fluorescein diacetate (FDA, Sigma Aldrich, USA) and propidium iodide (PI, Sigma Aldrich, USA) are two fluorescent dyes that allow for live and dead cell discrimination. 


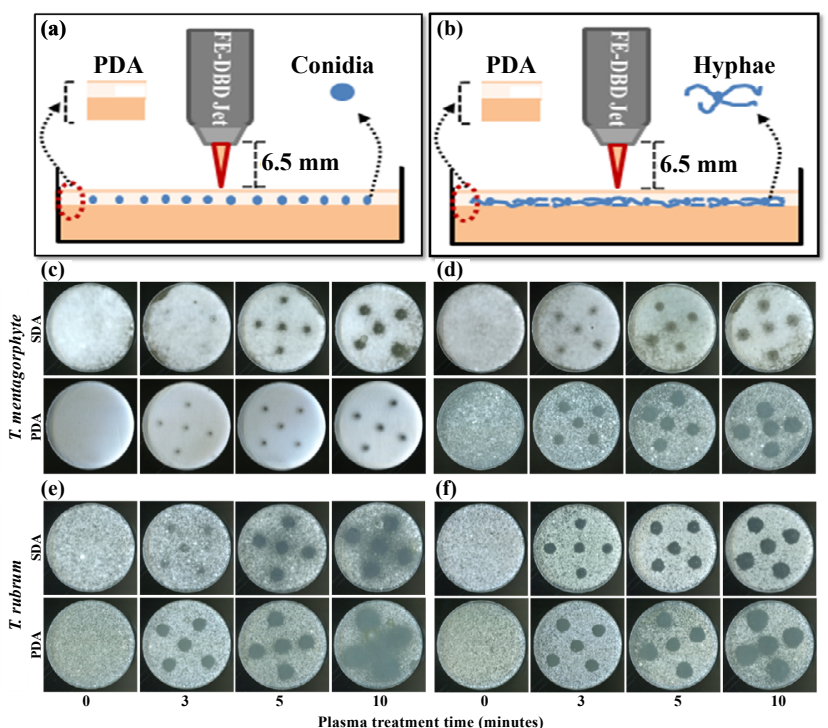

Figure 3: Growth inhibition of fungal conidia and hypha in an infected skin model as a result of plasma treatment. (a) Schematic diagram of the treatment conditions for fungal conidia and (b) hypha. (c) Growth inhibition images for fungal conidia and (d) hypha of T. mentagorophyte. (e) Growth inhibition images for fungal conidia and (f) hypha of $T$. rubrum. The samples were treated with plasma for $0,3,5$, and 10 minutes.

Live cells take up the non-fluorescent FDA and convert it to the green fluorescent metabolite fluorescein. This conversion is esterase dependent and serves as a live cell indicator. On the other hand, PI is a nuclei staining dye that can only pass through disordered areas of dead cell membranes to interact with and stain cell deoxyribonucleic acid (DNA).

All samples were stained with FDA and PI according to the method of Jones et al. [14] with some modification, and then a threedimensional confocal microscope was used to analyze the live and dead fungal conidia and hypha in the infected skin mimicking model. Briefly, the samples were treated with $1 \mu \mathrm{l}$ of FDA $(10 \mathrm{mg} / \mathrm{ml}$ stack solution) for 30 minutes at room temperature (RT). Then, the samples were washed with phosphate buffer saline (PBS, well-gene, Korea) and were stained with $5 \mu \mathrm{l}$ of PI ( $5 \mathrm{mg} / \mathrm{ml}$ stack solution) for 10 minutes at RT. The stained fungal conidia and hypha were observed with a laser scanning confocal microscope (LSM 700, Carl Zeiss Microscopy $\mathrm{GmbH}$, Jena, Germany) using a 10x objective lens and fluorescence optics (excitation at $488 \mathrm{~nm}$ for FDA and at $530 \mathrm{~nm}$ for PI; emission at $520 \mathrm{~nm}$ for FDA and at $620 \mathrm{~nm}$ for PI). The three-dimensional images of the fungal conidia and hyphae were analyzed using the ZEN imaging software (ZEN 2009, Carl Zeiss MicroImaging GmbH, Jena, Germany).

\section{Detection of intracellular ROS in fungal conidia after plasma treatment}

A calorimetric method was used to investigate intracellular ROS according to the method of Panngom et al. [15]. Briefly, the plasmtreated fungal conidia were centrifuged at $5,000 \mathrm{rpm}$ for 5 minutes, and the pellet was labeled with the oxidation-sensitive florescent dye 5-(6)-carboxy- $2^{\prime}, 7^{\prime}$-dichlorodihydrofluorescein diacetate (carboxy- $\mathrm{H}$ CFDA) dye (1:4 ratios in saline) for 30 minutes at $25^{\circ} \mathrm{C}$. Following incubation, the samples were centrifuged, and the pellet was mixed with $1 \mathrm{ml}$ PBS. Finally, $200 \mu \mathrm{l}$ of each sample was transferred into 96 well plates (black plate, clear bottom, corning incorporated USA) to measure fluorescence using a microplate reader (BioTek, synergy HT) with excitation at $485 \mathrm{~nm}$ and emission at $528 \mathrm{~nm}$ wavelengths.

\section{Statistical analysis}

The quantitative data were expressed as the mean \pm standard deviation (SD), and statistical comparisons were carried out with a Student's t-tests. Significant differences were indicated by $p<0.05$.

\section{Results and Discussions}

Fungi in general are responsible for various life threatening diseases in human such as septicemia, pneumonia, and systemic skin diseases [12]. T. rubrum and T. mentagrophytes are two major pathogens responsible for hair, nail, and skin disease. In particularly, people with immune deficiencies are more susceptible to fungal infections $[16,17]$. Therefore, fungal inhibition or removal is essential in medicine, and we need to develop techniques that are more effective in inactivating microorganisms but also less damaging to the patient.

Recently, plasma has attracted much interest in various fields because it has been found to have an antimicrobial nature, while also presenting other characteristics such as a low temperature, time efficiency, and non-toxic or selectively toxic properties $[18,19]$. However, the mechanism for the microbial inhibition of the plasma is not yet fully understood and needs further investigation. Detailed information on the mechanism for microbial inhibition would be of substantial clinical importance and would allow optimizing the effects of the plasma for various medical applications. Inactivation demands pronounced antimicrobial potential, but on the other hand, the surrounding tissue requires more of an influence on cellular regulation. Plasma needs to have high antimicrobial efficiency in vivo for antiseptic purposes, to inactivate large amounts of fungal growth, and to reduce or eliminate other forms of bio-burden.

As an initial step, the antifungal effects of the FE-DBD plasma jet that we developed were evaluated against two clinical isolates of dermatophytes, T. mentagrophytes and T. rubrum in vitro. Figures $2 \mathrm{a}$ and $2 \mathrm{~b}$ present the fungal cell viability in percentage and log scales when conidia were treated by FE-DBD plasma in $0.85 \%$ saline. We found that there was a time-dependent inhibitory effect the plasma on the conidia of both fungal strains. The inhibition rate of the conidia was measured by counting the CFU after 7 days of incubation at appropriate conditions. The conidia of both fungal strains failed to recover even when grown in normal conditions after exposure to the plasma, which might be evidence of the antifungal activity of the proposed plasma device. The inhibitory effect of the plasma started to be noticeable at low doses, i.e., exposure for 5 minutes, for both T. mentagrophytes and T. rubrum, and significant effects were observed at higher doses, i.e., after 10 minutes, as shown in Figure $2 \mathrm{a}$ and $2 \mathrm{~b}$. In particular, the antifungal effect of the 5 minute treatment were measured to be of about $96 \%(\mathrm{p}<0.003)$ for $T$. mentagrophytes and $90 \%(\mathrm{p}<0.016)$ for $T$. rubrum. This result indicates that T. mentagrophytes seems to be more susceptible to plasma than T. rubrum, and the treatment caused almost complete inhibition within 30 minutes.

Although, our plasma presented significant antifungal activities in the conidia of both strains, we need to evaluate a more complex and protected system to confirm the possibility of further study. Therefore, we designed a skin model infected with the conidia or hypha state of the fungus using agar, as shown in Figure $3 a$ and $b$. The dermatophytes are imperfect fungi that may form conidia by budding or by converting their existing parts. So, we treated both fungal conidia and hypha with plasma. In this study, fungal conidia were suspended in liquid PDA 
(a)

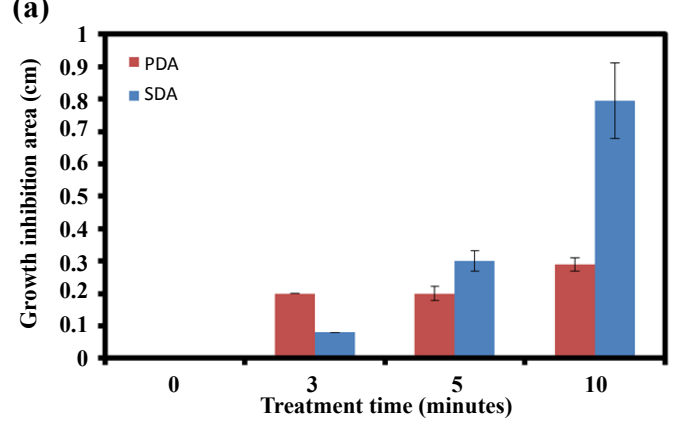

(c)

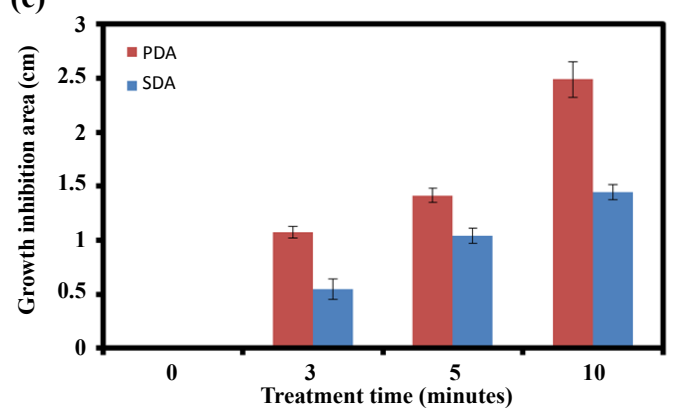

(b)

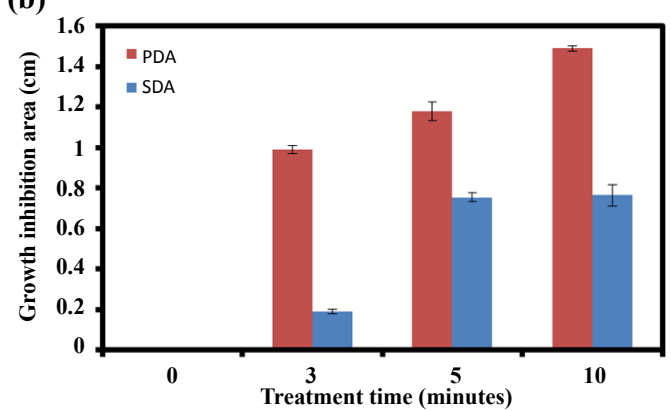

(d)

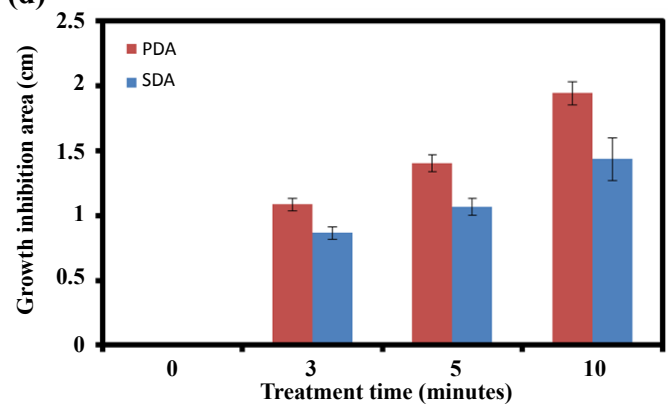

Figure 4: Size of the growth inhibition area in the infected skin model resulting from the plasma treatment. (a) Size of the growth inhibition area for fungal conidia and (b) for fungal hypha of T. mentagorophyte. (c) Size of the growth inhibition area for fungal conidia and (d) for fungal hypha of T. rubrum.

and SDA and were then spread on already prepared complete PDA and SDA culture plates, respectively. The hypha development samples were incubated for 3 days in appropriate conditions before treatment, and the conidia samples were treated with plasma at 5 different points. We found that plasma treatment inhibited fungal growth on the contacted area. The small colorless circles on the PDA and the SDA plates seen in Figure $3 \mathrm{c}-3 \mathrm{f}$ are the plasma-treated areas that show the growth inhibition regions. The diameter of these regions increases considerably as the treatment time increases in all conditions for both strains, as shown in Figure 4. In this infected skin mimicking model, T. rubrum seems to be more susceptible to plasma than T. mentagrophytes. This result reveals that besides fungal inactivation, a larger inhibition area may also be achieved with higher doses of plasma. Moreover, the plasma may penetrate deeper and may inactivate the fungus, a serious issue for treating skin and nail infections with topical antibiotics.

The literature shows fungi may develop differently on different media [20], and such differences may interfere with their inactivation. To evaluate the plasma efficacy of fungal inactivation on different mediums, we used PDA and SDA and found that plasma can reliably achieve inactivation of fungal growth in both cases in a defined area, even with does lower than 3 minutes. This shows that plasma is highly effective and can eradicate fungal conidia and hypha even in their preferred growth medium.

To visualize the effect of the plasma on fungal conidia and hypha, a fluorescence-based live-dead cell assay was performed via threedimensional confocal microscopy. Figure 5 shows the horizontally titled three-dimensional confocal images of the conidia and hypha of $T$. mentagorophyte located inside agar staining with FDA and PI. The FDA penetrates the live cells and converts to the green fluorescent metabolite fluorescein by the esterase reaction, as observed in untreated conidia and hypha samples shown in Figure $5 \mathrm{a}$ and $5 \mathrm{c}$. However, after plasma treatment for 3 minutes, both conidia and hypha nuclei were stained with PI, which can only pass through disordered areas of damaged cell membranes, regardless of their location inside the agar, as shown in Figure $5 \mathrm{~b}$ and $5 \mathrm{~d}$. This may be evidence of cell membrane disruption as a result of the plasma treatment. The thickness of the samples with fungal conidia and hypha $(\sim 40 \mu \mathrm{m})$ can be observed in the horizontally titled three-dimensional images.

Basically plasma is the mixture of ions, radicals, atoms and molecules which play critical roles in cellular signaling and microbial inactivation. Therefore, we measured OES by using a CCD spectrometer that can obtain emission spectra from wavelengths of 200 to $1100 \mathrm{~nm}$ in order to identify possible excited chemical species from plasma. Figure 6 demonstrates the optical emission spectra of the proposed FEDBD plasma jet to identify various excited species that are produced. The OES spectrum demonstrated that the hydroxyl radical $\left(\mathrm{OH}^{-}\right)$ appeared at a wavelength $308.9 \mathrm{~nm}(2 \Sigma-2 \Pi)$ [21] and that excited argon atoms (2p-1s) [22] were emitted between the range of 700 and $900 \mathrm{~nm}$ wavelength.

The observed spectra and the fungal inactivation confirm that plasma possesses antimicrobial properties since reactive species $[23,24]$, including hydroxyl radical, were observed to be present. The hydroxyl radical has high oxidation potential, which makes it a strong disinfectant [25]. The hydroxyl radical may react with ambient air and humidity to collectively affect the cells and result in fungal damage. So, the FE-DBD plasma jet allows an indirect flux of charged particles into the sample, including electrons and positive and negative ions, resulting in formation of radicals that interact with the fungal conidia or hypha. These radicals, ions, and UV sources can damage the cell membrane or organelles and may cause microbial etching and erosion 
Citation: Ali A, Hong YJ, Park J, Lee SH, Choi EH, et al. (2014) A Novel Approach to Inactivate the Clinical Isolates of Trichophyton mentagrophytes and Trichophyton rubrum by Using Non-Thermal Plasma. J Microb Biochem Technol 6: 314-319. doi:10.4172/1948-5948.1000161

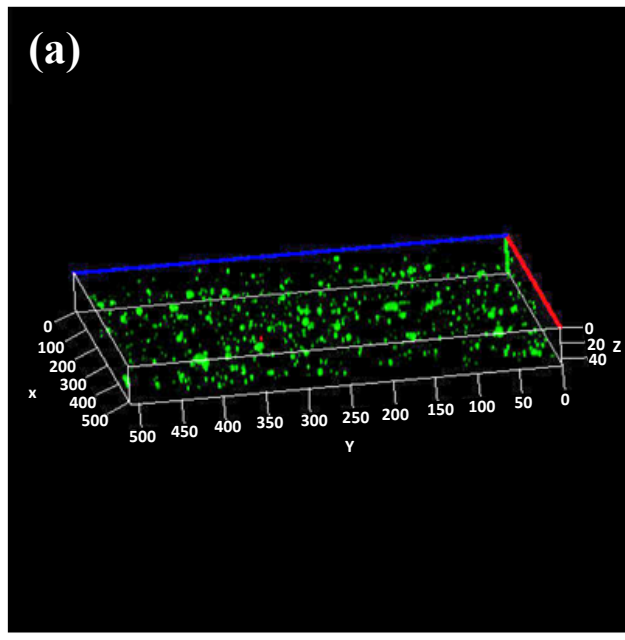

(c)

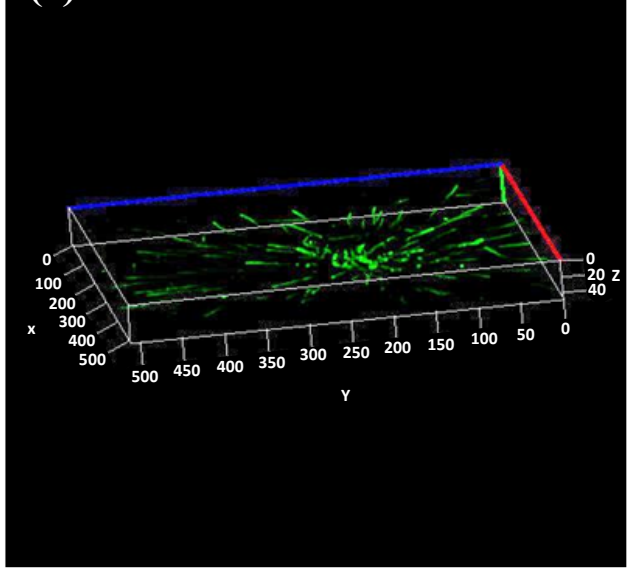

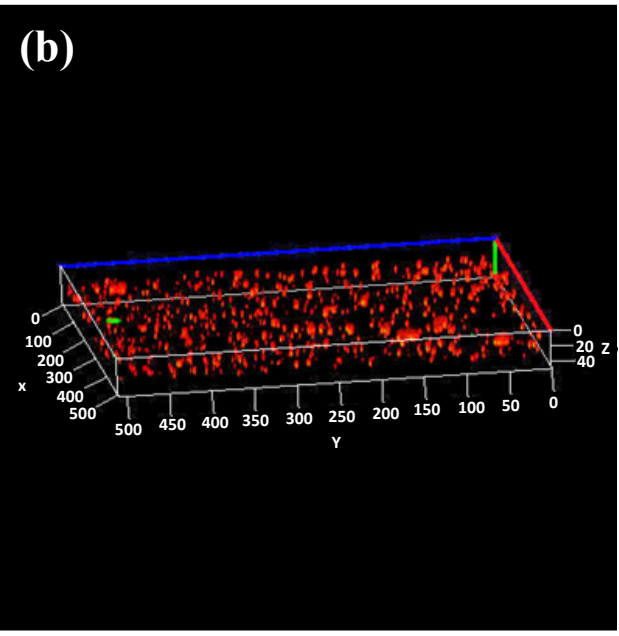

(d)

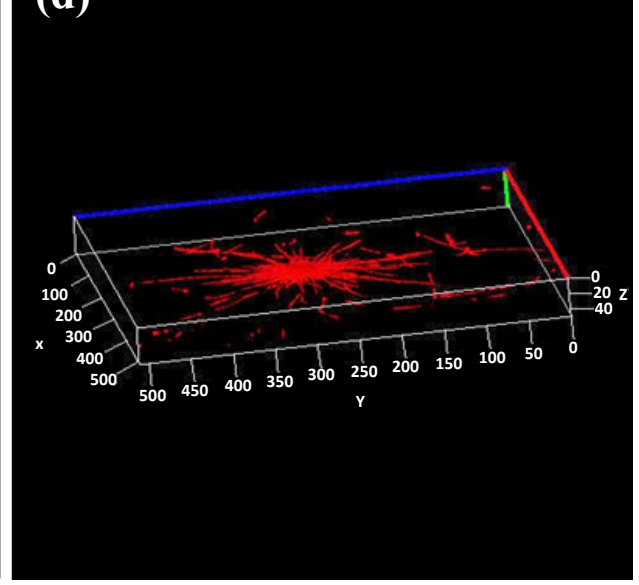

Figure 5: Three-dimensional confocal microscopic images of fungal conidia and hypha of $T$. mentagorophyte after treatment with plasma. (a) Fungal conidia for control. (b) Fungal conidia threated with plasma for 3 minutes. (c) Fungal hypha for control. (b) Fungal hypha threated with plasma for 3 minutes. Plasma-treated samples were stained with FDA and PI and three-dimensional images of the fungal conidia and hypha were taken using a laser scanning confocal microscope (LSM 700) with a 10x objective lens and fluorescence optics (excitation at $488 \mathrm{~nm}$ for FDA and at $530 \mathrm{~nm}$ for PI, and emission at $520 \mathrm{~nm}$ for FDA and at $620 \mathrm{~nm}$ for PI). The three-dimensional images were analyzed using the ZEN imaging software.

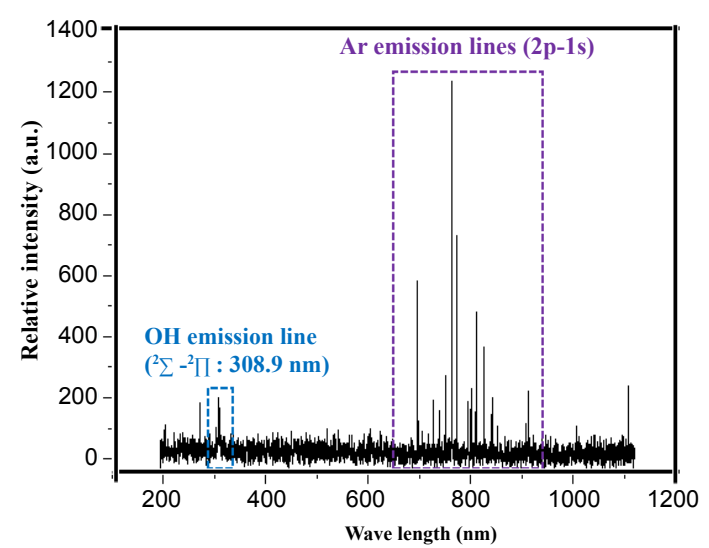

Figure 6: Optical emission spectrum (OES) of the FE-DBD plasma jet. A typical OES collected from the FE-DBD plasma jet in an air condition at $8 \mathrm{kV}$ power with spectral lines labeled according to their corresponding species. The $y$-axis represents the relative intensity in arbitrary units (a.u.), while x-axis represents the wavelength in $\mathrm{nm}$.
[26]. Therefore, we treated the fungal conidia with the same plasma doses as in quantitative treatments and measured intracellular ROS to investigate the correlation between plasma-generated reactive species and their antifungal effects.

In this experiment, we found a significant increase in the intracellular ROS in the conidia of both strains after plasma treatment for 5 minutes, and then the increased intracellular ROS level started to decrease, probably as a result of the decline in fungal viability, as shown in Figures 2 and 7. The intracellular ROS levels of T. mentagorophyte conidia treated with plasma were measured to be $268 \%$ after 5 minutes, $146 \%$ after 10 minutes, $24 \%$ after 20 minutes, and $11 \%$ after 30 minutes, and the intracellular ROS levels of T. rubrum conidia treated with plasma were measured to be $148 \%$ after 5 minutes, $121 \%$ after 10 minutes, $89 \%$ after 20 minutes, and $41 \%$ after 30 minutes, as compared to control (Figure $5 \mathrm{a}$ and $5 \mathrm{~b}$ ). These results assumed that the sudden decline in the intracellular ROS resulted from the reduction in live cells due to plasma treatment, as shown in Figure 2a and $2 \mathrm{~b}$. 
Citation: Ali A, Hong YJ, Park J, Lee SH, Choi EH, et al. (2014) A Novel Approach to Inactivate the Clinical Isolates of Trichophyton mentagrophytes and Trichophyton rubrum by Using Non-Thermal Plasma. J Microb Biochem Technol 6: 314-319. doi:10.4172/1948-5948.1000161

(a)

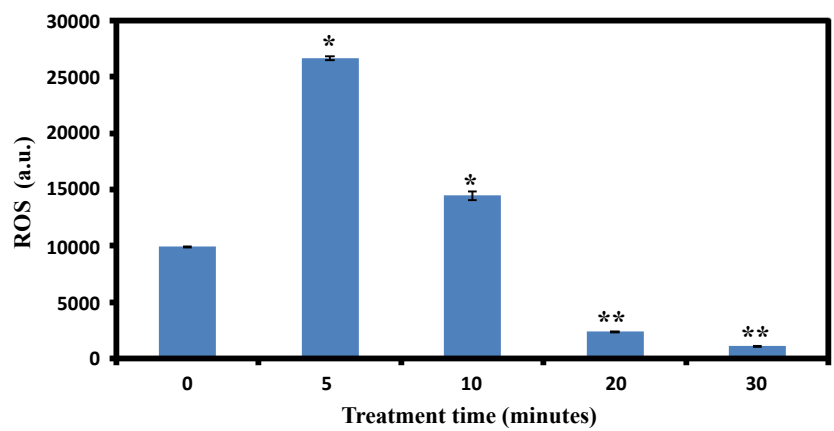

(b)

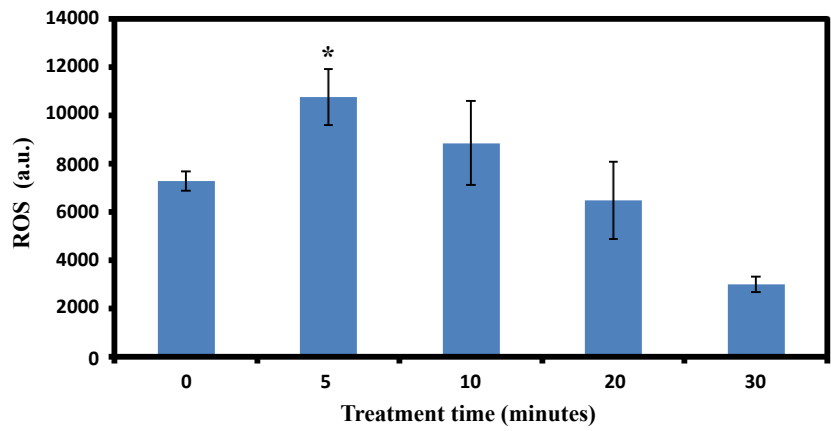

Figure 7: Intracellular reactive oxygen species (ROS) detected in the conidia of (a) $T$. mentagorophyte and (b) for $T$. rubrum. The $x$-axis represents the time for the plasma treatment while they $y$-axis represents the intracellular ROS intensity in arbitrary units (a.u.). All data are expressed as a mean \pm standard deviation and were analyzed using Student's t-tests. Statistical significance was considered as $p<0.05\left({ }^{*} p<0.05 ;{ }^{* *} p<0.005\right.$ vs. control at the same time)

\section{Conclusion}

In this study, we used an in vitro skin model to test a novel approach for inactivation of dermatophytes. We demonstrated that an FEDBD plasma jet has considerable antifungal potential against clinical isolates of the most frequently encountered causative pathogens of dermatophytosis, T. mentagorophyte and T. rubrum. Their conidia and hypha were inactivated within 3 minutes of plasma treatment, even inside the agar or in liquid. In addition, we found a correlation between the intracellular ROS level and the cell viability depending on the plasma treatment time, which suggests that plasma played a critical role in generating reactive species for fungal inactivation.

Our findings suggest there is a possibility to use non-thermal plasma to treat dermatophytosis. In the future, plasma may serve as a supportive and/or alternative therapy for fungal related infections, giving an additional choice for physicians choosing a treatment for their patients.

\section{Acknowledgement}

This work was supported by the National Research Foundation of Korea (NRF-2010-0027963), funded by the Korean government (MSIP) .

\section{References}

1. Havlickova B, Czaika VA, Friedrich M (2008) Epidemiological trends in skin mycoses worldwide. Mycoses 51 Suppl 4: 2-15.

2. Weitzman I, Summerbell RC (1995) The dermatophytes. Clin Microbiol Rev 8: 240-259.

3. Male O (1990) The significance of mycology in medicine. In: Hawksworth DL (ed), Frontiers in Mycology. Wallingford: CAB International 131-156.
4. Huang DB, Ostrosky-Zeichner L, Wu JJ, Pang KR, Tyring SK (2004) Therapy of common superficial fungal infections. Dermatol Ther 17: 517-522.

5. Tejasvi T, Sharma VK, Sethuraman G, Singh MK, Xess I (2005) Invasive dermatophytosis with lymph node involvement in an immunocompetent patient. Clin Exp Dermatol 30: 506-508.

6. Zapata Garrido AJ, Romo AC, Padilla FB (2003) Terbinafine hepatotoxicity. A case report and review of literature. Ann Hepatol 2: 47-51.

7. Fridman G, Friedman G, Gutsol A, Shekhter AB, Vasilets VN, et al. (2008) Applied plasma medicine. Plasma Process Polym 5: 503-533.

8. Iza F, Kim GJ, Lee SM, Lee JK, Walsh JL, et al. (2008) Microplasmas: sources, particle kinetics and biomedical applications. Plasma Process Polym 5: 322344.

9. Fridman G, Brooks AD, Balasubramanian M, Fridman A, Gutsol A, et al. (2007) Comparison of direct and indirect effects of non-thermal atmospheric-pressure plasma on bacteria. Plasma Process Polym 4: 370-375.

10. Gostev VA, Ignakhin VS, Popova EK, Ostashkov OA (2008) Cold plasma-a powerful agent for biological applications. In: Gu"ceri S, Fridman A, editors. Plasma assisted decontamination of biological and chemical agents. Dordrecht Springer Science, Business Media BV: 65.

11. Stoffels E, Kieft IE, Sladek REJ, van den Bedem LJM, van der Laan Ep, et al. (2006) Plasma needle for in vivo medical treatment: recent developments and perspectives. Plasma Sources Sci Technol 15: 169-180.

12. Rippon JW (1988) Medical Mycology- the Pathogenic Fungi and the Pathogenic Actinomycetes (3rdedn), W. B. Saunders Company, Philadelphia.

13. Liu P, Luo L, Guo J, Liu H, Wang B, et al. (2010) Farnesol induces apoptosis and oxidative stress in the fungal pathogen Penicillium expansum. Mycologia 102: 311-318.

14. Jones KH, Senft JA (1985) An improved method to determine cell viability by simultaneous staining with fluorescein diacetate-propidium iodide. J Histochem Cytochem 33: 77-79.

15. Panngom K, Lee SH2, Park DH3, Sim GB1, Kim YH4, et al. (2014) Non-thermal plasma treatment diminishes fungal viability and up-regulates resistance genes in a plant host. PLoS One 9: e99300.

16. Galgiani JN, Ampel NM (1990) Coccidioidomycosis in human immunodeficiency virus-infected patients. J Infect Dis 162: 1165-1169.

17. Wheat LJ, Connolly-Stringfield PA, Baker RL, Curfman MF, Eads ME, et al. (1990) Disseminated histoplasmosis in the acquired immune deficiency syndrome: clinical findings, diagnosis and treatment, and review of the literature. Medicine (Baltimore) 69: 361-374.

18. Panngom K, Baik KY, Nam MK, Han JH, Rhim H, et al. (2013) Preferential killing of human lung cancer cell lines with mitochondrial dysfunction by nonthermal dielectric barrier discharge plasma. Cell Death Dis 4: e642.

19. Park BJ, Lee DH, Park JC, Lee IS, Lee KY, et al. (2003) Sterilization using a microwave-induced argon plasma system at atmospheric pressure. Phys Plasmas 10: 11.

20. Oyeka CA (2000) Trichophyton mentagrophytes a keratinophilic fungus Revista Iberoamericana de Micología Apdo, Spain.

21. Dieke GH, Crosswhite HM (1961) The ultraviolet bands of $\mathrm{OH}$ Fundamental data. J Quant Spectrosc Radiat Transfer 2: 97-199.

22. Wiese WL, Smith MW, Miles BM (1969) Atomic Transition Probabilities. NTIS 2: $187-224$.

23. Moisan M, Barbeau J, Moreau S, Pelletier J, Tabrizian M, et al. (2001) Lowtemperature sterilization using gas plasmas: a review of the experiments and an analysis of the inactivation mechanisms. Int J Pharm 226: 1-21.

24. Laroussi M (2002) Nonthermal decontamination of biological media by atmospheric-pressure plasmas: review, analysis, and prospects. IEEE Trans Plasma Sci 30: 4.

25. Grabowski LR (2006) Pulsed Corona in Air for Water Treatment. PhD Thesis, Eindhoven University of Technology, Eindhoven, Netherland.

26. Moisan M, Barbeau J, Crevier MC, Pelletier J, Philip N (2002) Plasma sterilization. Methods and mechanisms. Pure Appl Chem 74: 349-358. 